Research Article

\title{
Seroprevalence of Taenia solium and Trichinella spiralis among Humans and Pigs in Ghana
}

\author{
Henry Ofosu Addo (D), ${ }^{1}$ Ayodele O. Majekodunmi (iD, ${ }^{2}$ Eric Sampane-Donkor (D), ${ }^{3}$ \\ Lawrence Henry Ofosu-Appiah, ${ }^{4}$ David Opare, ${ }^{4}$ Godfred Owusu-Okyere, ${ }^{1}$ \\ Kingsley Ebenezer Amegah $\mathbb{D}^{5},{ }^{5}$ and Langbong Bimi ${ }^{1}{ }^{1}$ \\ ${ }^{1}$ Department of Animal Biology and Conservation Science, University of Ghana, Ghana \\ ${ }^{2}$ Livestock and Poultry Research Centre, College of Basic and Applied Sciences, University of Ghana, Ghana \\ ${ }^{3}$ Department of Medical Microbiology, University of Ghana, Ghana \\ ${ }^{4}$ National Public Health Reference Laboratory, Korle-Bu, Ghana \\ ${ }^{5}$ Hohoe Municipal Hospital, Ghana Health Service, Ghana
}

Correspondence should be addressed to Langbong Bimi; lbimi@ug.edu.gh

Received 29 May 2021; Accepted 25 August 2021; Published 8 October 2021

Academic Editor: Klaus Wimmers

Copyright (c) 2021 Henry Ofosu Addo et al. This is an open access article distributed under the Creative Commons Attribution License, which permits unrestricted use, distribution, and reproduction in any medium, provided the original work is properly cited.

\begin{abstract}
In this study, the seroprevalence of the intestinal worms Taenia solium and Trichinella spiralis in humans and pigs was assessed. A cross-sectional serological study design was performed. Blood samples were collected from 322 humans and 245 pigs used in the study. These were tested for markers of antibodies for Taenia solium and Trichinella spp. Demographic data such as sex, age, education, pig farming practices, and water source used were also obtained. An overall seroprevalence of $3.1 \%$ was recorded for Taenia solium in humans. There was also a statistical association between pig management system employed by pig farmers and seropositivity to Taenia solium $(p=0.005)$. Factors such as mode of waste disposal $(p=0.003)$ and water source used statistically correlated with Taenia solium seroprevalence among humans. For the pig samples, a Taenia solium seroprevalence of $24.9 \%$ was recorded. All the pig samples which tested positive for Taenia solium were reared on the free-ranged system. This study also recorded a seroprevalence of $0.31 \%$ for Trichinella spp. for humans and a seroprevalence of $4.5 \%$ for Trichinella spp. for pigs. Again, all the samples that showed serological evidence of Trichinella spp. among pigs came from those pigs which were raised on the free-ranged system. Proper pig management practice is a very important tool for controlling these intestinal parasites in both humans and animals. This study recommends public health education among the general public and good pig farming practices.
\end{abstract}

\section{Introduction}

Zoonotic enteric parasites are universal, presenting a significant public health challenge to human beings primarily due to an intimate relationship with domestic animals and wildlife and inadequate water, sanitation, and hygiene [1]. Pigrelated zoonoses such as Taenia solium and Trichinella spiralis are implicated in areas with poor hygiene, water, and sanitation issues $[2,3]$. These pig-related zoonotic diseases mostly affect people of the lower socioeconomic class, mostly in developing countries [4]. Taenia solium infections have been reported in several West African countries, although official statistics are often lacking [5]. Pork consumption has been recorded as a risk factor for Taenia solium and Trichinella infections [2]. Data on the prevalence of porcine Taenia solium are extremely scarce, unreliable, or underestimated in West Africa [5,6]. Melki et al. [6] further argued that the available statistics are typically based on data obtained from "official" abattoirs and slaughterhouses, but in most countries in the developing world, majority of pigs 
are not slaughtered within approved slaughterhouses but often clandestinely without veterinary supervision. Coupled with that, the massive use of the extensive form of pig farming increases the exposure of pigs to contracting viable Taenia solium eggs $[4,7]$. Lack of or inadequate meat inspections especially in unregistered pig slaughterhouses usually result in contaminated pork or pork products on the open markets [8]. However, Zoli et al. [5] argued that data about porcine cysticercosis obtained from pig slaughterhouses should be interpreted with caution. They argued that the data obtained from these meat inspections in these slaughterhouses are not representative of the real situation, since a large proportion of pigs, and certainly the cysticercotic pigs, are slaughtered outside the pig slaughterhouses [5]. In Ghana, some pigs are slaughtered either at home or on the farm and not at the pig slaughterhouse. There is also a general acceptance that pigs sent for slaughter are screened by tongue inspection by veterinarians, resulting in higher apparent prevalence on pig farms as compared to slaughterhouses [9]. According to Braae et al. [9], this approach causes bias issues in the studies done at slaughterhouses, which will underestimate the true prevalence of porcine cysticercosis, as pigs which carry high-intensity infections might have been eliminated from the sample. According to Onah and Chiejina [10], meat inspection usually underestimates the true prevalence of porcine cysticercosis where they found $20.5 \%$ of pigs with cysticercosis after detailed inspection of the carcass where the official figure of meat inspection reported only $3 \%$ of porcine cysticercosis. The detection of porcine cysticercosis usually based on the inspection of meat and tongue palpation is characterized by very low sensitivity which can lead to the underestimation of the true prevalence of porcine cysticercosis $[11,12]$. For instance, in Burkina Faso, the first records of porcine cysticercosis around the year 2000 were based on meat inspection and a very low prevalence of $0.57 \%$ was recorded; however, later in 2011, a seroprevalence of $39.6 \%$ was recorded based on Ag-ELISA [13]. Bimi et al. [2] postulated that pork handlers and their households, together with people who prepare pork, stand a higher risk of tapeworm infection. From their study, men who were the pork sellers and their immediate family members stood a greater risk of infection. Occupational exposure is the reason why slaughterhouse workers and pork sellers who handle the offals and come into contact with the pig/pork stand a higher chance of contracting pig-related zoonoses like Taenia solium and Trichinella spp. when contaminated or raw meat is consumed. Studies on helminth infections among humans in Ghana are inadequate, especially among such groups as pig farmers, slaughterhouse workers, and pork sellers who are considered important players in the handling of pig/pork. Very few studies also exist on Taenia solium among pigs in Ghana, but these studies are concentrated on postmortem identification of viable cysts which might underestimate the true prevalence. Also, data on how the pigs were raised whether free-ranged or confined are not stated [2, 14, 15]. Despite its presence in several African countries and with the increase in pork consumption in Ghana, data on trichinellosis in humans is limited in Ghana. There has been no study in Ghana which has positively recorded Trichinella infections among humans. Also, not much studies have been done on the prevalence of Trichinella in pigs in Ghana, although Permin et al. [16] found no Trichinella larvae among cross-bred pigs in the Upper East Region of Ghana. This study was therefore conducted to determine the seroprevalence of these intestinal worms (Taenia solium and Trichinella spiralis) in humans in occupational exposure to pigs and also in pigs raised in the both the free-ranged and confined production systems.

\section{Methods}

2.1. Study Area. The study area purposively selected for this study has been noted as places of rigorous pig rearing and trading activities. Places selected were Ga South Municipality, Accra Metropolitan Assembly (AMA), Ladadekotopon Municipality, and Ledzokuku Municipality. These places are located along the Coast of the Greater Accra Metropoli$\tan$ Area (GAMA) (Figure 1). These places have pigs housed in makeshift pens and mostly practice the free-ranged system of pig production. The Upper East Region located in the Northern part of Ghana was also purposively included in this study because it serves as a major conduit of pigs supplied to Southern Ghana (Figure 2).

2.2. Study Design. The study adopted a cross-sectional, serological study design in collecting data. The study period was between January and March 2018 among study participants from Accra who were pig farmers, slaughterhouse workers, pork sellers, and also among people living in adjoining households to pig farms. Serological studies were also done on pigs found within the selected communities during the same period. A similar cross-sectional serological survey was conducted among humans and pigs in the Upper East Region between July and August 2018.

2.3. Study Population. Groups which were involved in the pig/pork trade in Ghana were purposively selected for this study. The selected groups were identified as key players in the pig or pork trade in Ghana. The groups were pig farmers, slaughterhouse workers, and pork sellers. These were done to determine occupational exposure to pigs to determine the seroprevalence to the intestinal worms. To determine nonoccupational exposure to Taenia solium and Trichinella spiralis infections, households in close proximity to pig farms were recruited to be part of the study.

2.4. Sample Size and Selection Procedure. The calculation of the sample size was based on the formula put forward by Charan and Biswas [17]. The proportion assumed was $13.15 \%$ prevalence rate of pig-related zoonoses among those handling pig/pork as purported by Bimi et al. [2].

Thus, we have

$$
n=\frac{Z^{2} p(1-p)}{d^{2}}
$$

level $=1.96 ; p$ is the assumed proportion from similar studies which is $13.15 \%$ expressed in decimal $=0.1315$; 

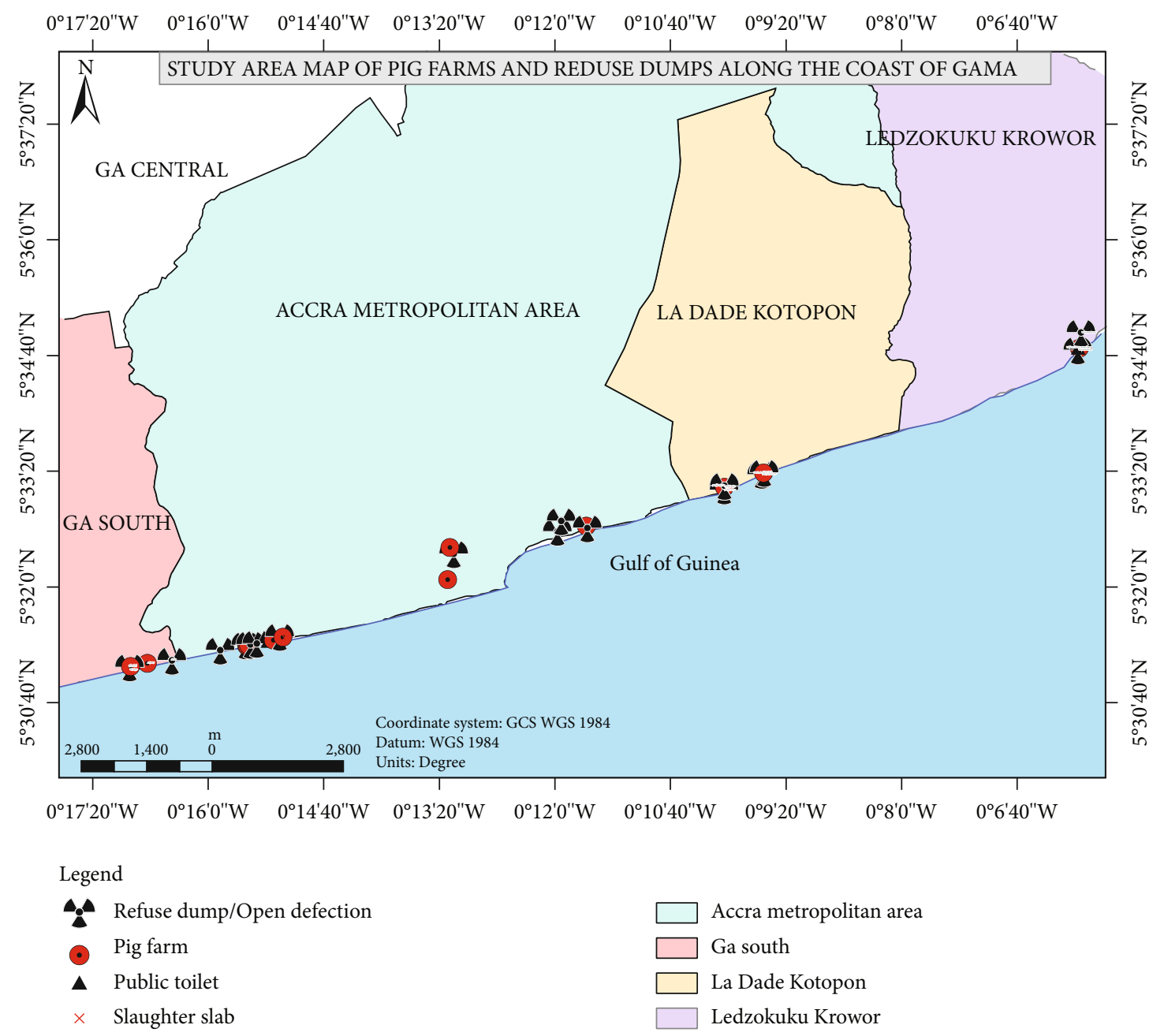

Figure 1: Map of Accra showing selected districts.

$q=1-p$ is the probability of the event not occurring, in this case $1-0.1315 ; d$ is the $5 \%$ margin of error expressed in decimal $=0.05$.

$$
\begin{aligned}
& n=\frac{1.96^{2} \times 0.1315(1-0.1315)}{0.05^{2}}=n=\frac{0.43874}{0.0025}=175.5, \\
& n \cong 176 .
\end{aligned}
$$

Adding a 5\% nonresponse rate, we have

$$
\begin{gathered}
\frac{5 \times 176=9}{100}, \\
n=176+9=185,
\end{gathered}
$$

for human samples for this study.

For the pig samples used for this study, the proportion assumed was $18.8 \%$ prevalence rate as purported by Bimi et al. [2].
Thus, we have

$$
\frac{n=Z^{2} p(1-p)}{d^{2}}
$$

level $=1.96 ; p$ is the assumed proportion from similar studies which is $18.8 \%$ expressed in decimal $=0.188 ; q=1-p$ is the probability of the event not occurring, in this case $1-0.188=0.812 ; d$ is the $5 \%$ margin of error expressed in decimal $=0.05$.

$$
\begin{aligned}
& n=\frac{1.96^{2} \times 0.188(1-0.188)}{0.05^{2}}=n=\frac{0.5864}{0.0025}=234.58, \\
& n \cong 235 .
\end{aligned}
$$

Adding a 5\% nonresponse rate, we have

$$
\begin{gathered}
\frac{5 \times 176}{100}=9, \\
N=235+9=244,
\end{gathered}
$$

where $\cong 245$ pig samples were sampled for this study. 


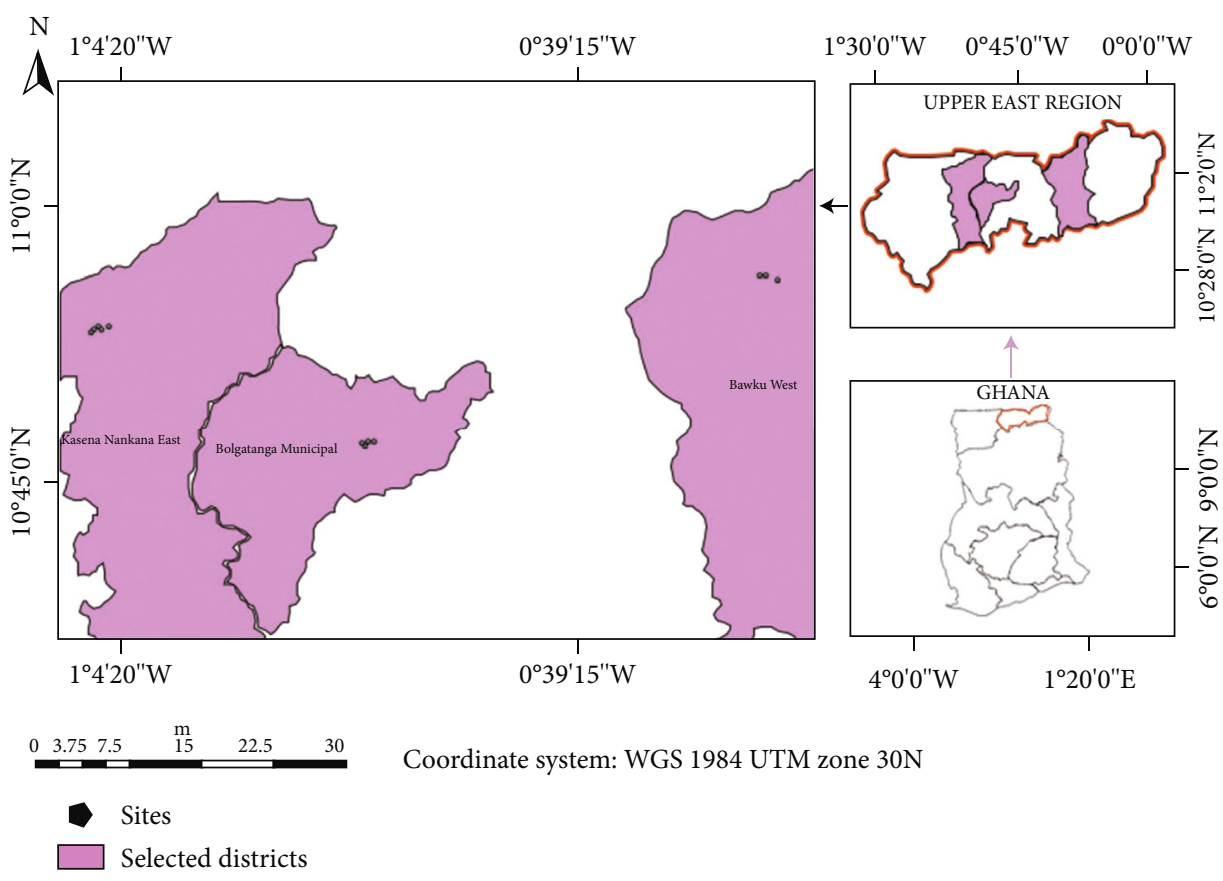

FIgURE 2: Map of the Upper East Region showing the selected study districts.

\subsection{Data Collection Methods}

2.5.1. Blood Sample Collection and Processing. Five (5) $\mathrm{ml}$ of blood samples was collected from both humans and pigs into the gel separation vacutainer tubes. The blood samples were appropriately labelled and transported on ice to the National Public Health Reference Laboratory, Korle-Bu, Ghana. There, the blood samples were allowed to clot and were centrifuged for 10 minutes. The serum was then placed in cryovials for processing.

2.5.2. Enzyme-Linked Immunosorbent Assay (ELISA). Samples collected for this study were serologically examined for the evidence of markers of Teania solium and Trichinella spiralis in both humans and pigs. The purpose of such a test was to detect serum antibodies or antibody-like substances that appear specifically in association with certain diseases.

The Novatec Immundiagnostica GMBH Taenia solium IgG (Lot number: TAE-061; sensitivity 94\%; specificity 95\%; Novatec Immundiagnostica, Germany) was the ELISA kit used for the serological testing of Taenia solium in humans, and porcine sera were analyzed for the detection of antibodies against Taenia solium (IgG) using the Novatec Vetline Taenia solium (Lot number: TAEVT-061-1; sensitivity 95\%; specificity 95\%; Novatec Immundiagnostica, Germany). The Novatec Immundiagnostica GMBH Taenia solium IgG is intended for the qualitative determination of IgG class antibodies against Taenia solium in human serum or plasma. The VetLine Taenia ELISA is also intended for the qualitative determination of IgG antibodies against Taenia in veterinary mammalian serum. The VetLine contains 12 break-apart 8-well snap-off strips coated with Taenia antigens. This ELISA kit makes use of the larval extract of pig tapeworm as an antigen, and this extract was fractionated.
The manufacturer's instructions were followed in testing serum samples for the presence of exposure to Taenia solium. Before assaying, all samples were diluted 1:100 with the IgG Sample Diluent.

The IBL International GMBH Trichinella spiralis IgG ELISA was the ELISA kit used for the serological testing of Trichinella spiralis in humans. The IBL International GMBH Trichinella spiralis IgG ELISA (Lot number: TRIG-065; sensitivity 100\%; specificity 95\%; IBL International, Germany) is intended for the qualitative determination of IgG class antibodies against Trichinella spiralis in human serum or plasma (citrate, heparin). The Trichinella spiralis ELISA contains 12 break-apart 8-well snap-off strips coated with Trichinella spiralis. The manufacturer's instructions were followed in testing serum samples for the presence of exposure to Trichinella spiralis. Before assaying, all samples were diluted 1:100 with the IgG Sample Diluent.

Serum samples from pigs also were analyzed for the detection of antibodies against Trichinella spiralis (IgG) using the PrioCHECK Porcine Trichinella Ab 450 (Lot number: T170301L; sensitivity 97\%; specificity 98\%; Prionics, Switzerland). The PrioCHECK Trichinella $\mathrm{Ab}$ is used for in vitro detection of IgG antibodies against Trichinella spp. in serum and meat juice of pigs. The manufacturer's instructions were strictly followed in testing pig serum samples for the presence of exposure to Trichinella spp. The following steps were applied:

(a) Sample dilution: the control sample was reconstituted by adding $150 \mu \mathrm{l}$ of demineralized water. This was mixed by vortexing thoroughly and inverting the vial several times. A dummy plate was used for the first sample dilution. The positive controls were designated for wells A1 and B1 of the dummy plate 
to which $10 \mu \mathrm{l}$ of these positive controls was added. Weak positive controls were assigned to wells C1 and D1 of the dummy plate and to which $10 \mu \mathrm{l}$ of the weak positive controls was added. Ten (10) $\mu$ l of negative controls was added to wells E1 and F1 of the dummy plate. Ten (10) $\mu$ l of serum samples was then added to the remaining wells of the dummy plate. Ninety (90) $\mu \mathrm{l}$ of sample diluent was added to each well of the dummy plate, and it was mixed by pipetting up and down for 5 times. To the test plate, $80 \mu \mathrm{l}$ of the sample diluent was added to each well. Twenty (20) $\mu \mathrm{l}$ of the diluted samples and $20 \mu \mathrm{l}$ of the controls were transferred from the dummy plate to the test plate and mixed by pipetting up and down 5 times

(b) Sample incubation: the samples were then incubated on the test plate for $30 \pm 1$ minutes at room temperature $\left(22^{\circ} \mathrm{C} \pm 3\right)$. The test plate was washed four times with $300 \mu \mathrm{l} 1 \mathrm{x}$ wash fluid working solution using the Biotek Microplate plate washer

(c) Conjugate incubation: the conjugate was first diluted before use by the proportion of $400 \mu \mathrm{l}$ of conjugate to $11.6 \mathrm{ml}$ conjugate diluent for one full plate of 96 wells. One hundred (100) $\mu \mathrm{l}$ of the diluted conjugate was added to each well of the test plate. The test plate was afterwards incubated at $22^{\circ} \mathrm{C} \pm 3$ for $30 \pm 1$ minutes. The test plate was then washed four times with $300 \mu \mathrm{l} 1 \mathrm{x}$ wash fluid working solution using the Biotek Microplate plate washer

(d) Detection: one hundred (100) $\mu \mathrm{l}$ of the chromogen (TMB) substrate was added to each well on the test plate. The test plate was incubated for $15 \pm 1$ minutes at $22^{\circ} \mathrm{C} \pm 3$. One hundred (100) $\mu \mathrm{l}$ of the stop solution was added to each well of the test plate. The addition of the stop solution started $15 \pm 1$ minutes after the first well was filled with chromogen (TMB) substrate solution. The stop solution was added in the same order as the chromogen (TMB) substrate solution was dispensed. The colour of the positive controls changed from blue to yellow. The test plate was shaken shortly (5-10s) on an orbital shaker. The result of the test plate was read at $450 \mathrm{~nm}$ within 15 minutes by using a Biotek plate reader

For Taenia solium, results were interpreted as positive if it was greater than $11 \mathrm{NTU}(\mathrm{U})$. Positive results mean that antibodies against the pathogen were present. Results were interpreted as negative if it was less than 9 NTU (U). Thus, the sample contained no antibodies against the pathogen. For Trichinella spiralis, all results that were above or equal to the cut-off of $15 \mathrm{PP}$ were considered positive. Results recorded which were below the cut-off of 15 percentage positivity (PP) were considered negative.

2.6. Data Analysis. Questionnaire and laboratory data were entered into a spreadsheet (Excel; Microsoft, Redmond,
WA, USA) and analyzed using STATA software version 14.2 (StataCorp LP, USA). Descriptive measures such as the mean, frequencies, and percentages were employed to describe the variables under study. Prevalence was calculated as all individuals who showed serological evidence of infection over the total number tested as a percentage.

2.7. Ethical Considerations. The study protocol was approved by the Ethics Committee for Basic and Applied Sciences (ECBAS). This study collected data (blood samples) from both humans and pigs between December 2017 and August 2018. The study protocol (ECBAS 010/17-18) was carefully and verbally explained to each participant in a language they understood and each participant was assured of confidentiality. Each participant also had the chance to ask questions at each point of the study to which answers were provided. After voluntary agreement to participate, participants consented and were at liberty to withdraw from the study at any point of the study. Literate participants were given written forms to read and sign. Illiterate participants were asked to provide a literate witness who signed on their behalf before they were asked, in the presence of their witness to thumbprint the consent form.

\section{Results}

3.1. Seroprevalence of Taenia solium among Humans in Accra and Upper East. From the study, the overall seroprevalence for Taenia solium recorded for humans in both Accra and the Upper East Region was 3.1\% (10/322). However, there was no statistical relationship between the location of where the humans came from and seropositivity to Taenia solium and cysticercosis $(p=0.656)$ (Table 1$)$.

3.1.1. Age Group. Seropositivity to Taenia solium was not correlated with the age group in this study $(p=0.584)$. The highest seroprevalence was recorded among age groups 41-50 years and 51-60 years (Table 2).

3.1.2. Occupation. Occupationally related exposure to pigs such as slaughterhouse workers, pig farmers, and pork sellers had no statistical relationship with seropositivity to Taenia solium $(p=0.155)$ (Table 2 ).

3.1.3. Mode of Waste Disposal. The highest seroprevalence was recorded among respondents who practiced crude or open dumping (14.6\%). There was a statistical relationship between waste disposal and seropositivity to Taenia solium $(p=0.003)$ (Table 2).

3.1.4. Water Source Used. The water source used by respondents was statistically associated with Taenia seroprevalence $(p=0.025)$. All the respondents who tested positive for Taenia solium relied on community standpipe for their water use needs (6.2\%). Respondents who used other water sources all tested negative for Taenia solium (Table 2).

3.1.5. Pig Management System. Only pig farmers who raised pigs on the free-ranged system tested positive to Taenia solium in the Upper East. The highest Taenia solium 
TABLE 1: Seroprevalence of Taenia solium in humans and test of association between location and Taenia solium seropositivity.

\begin{tabular}{|c|c|c|c|c|c|c|c|}
\hline Location & $N(\%)$ & Positive (\%) & Negative (\%) & Prevalence (\%) & ToP & $z$ & Std. Err. \\
\hline Accra & $238(73.9)$ & $8(3.4)$ & $230(96.6)$ & 3.4 & 0.0336 & 0.45 & 0.011 \\
\hline Upper East & $84(26.1)$ & $2(2.4)$ & $82(97.6)$ & 2.4 & 0.0238 & & 0.017 \\
\hline Total & $322(100.0)$ & $10(3.1)$ & $312(96.9)$ & 3.1 & & & \\
\hline
\end{tabular}

Fisher's exact $=0.1983 . p$ value $=0.656 .{ }^{*}$ ToP: two-sample test of proportion.

TABle 2: Association between Taenia solium seropositivity and predictor variables.

\begin{tabular}{|c|c|c|c|}
\hline Variables & Negative (\%) & Positive (\%) & Chi-square ( $p$ value) \\
\hline \multicolumn{4}{|l|}{ Sex } \\
\hline Male & $209(95.9)$ & $9(4.1)$ & \multirow{2}{*}{$2.3467(0.126)$} \\
\hline Female & $103(99.0)$ & $1(1.0)$ & \\
\hline \multicolumn{4}{|l|}{ Age group (years) } \\
\hline Under 20 & $17(94.4)$ & $1(5.6)$ & \multirow{6}{*}{$3.7622(0.584)$} \\
\hline $20-30$ & $54(98.2)$ & $1(1.8)$ & \\
\hline $31-40$ & $99(98)$ & $2(2.0)$ & \\
\hline $41-50$ & $82(96.5)$ & $3(3.5)$ & \\
\hline $51-60$ & $41(93.2)$ & $3(6.8)$ & \\
\hline 61 and above & $19(100.0)$ & $0(0.0)$ & \\
\hline \multicolumn{4}{|l|}{ Occupation } \\
\hline Slaughterhouse worker & $26(100.0)$ & $0(0.0)$ & \multirow{4}{*}{$5.2332(0.155)$} \\
\hline Farmer & $131(97.0)$ & $4(3.0)$ & \\
\hline Community members & $97(94.2)$ & $6(5.8)$ & \\
\hline Pork seller & $58(100.0)$ & $0(0.0)$ & \\
\hline \multicolumn{4}{|l|}{ Mode of waste disposal } \\
\hline Collection trucks & $24(100.0)$ & $0(0.0)$ & \multirow{8}{*}{$26.8043 *(\mathbf{0 . 0 0 3})+$} \\
\hline Community collection point & $44(100.0)$ & $0(0.0)$ & \\
\hline Community refuse dump & $51(96.2)$ & $2(3.8)$ & \\
\hline Dumped into the sea & $47(97.9)$ & $1(2.1)$ & \\
\hline Household collection to community dumpsite & $9(100.0)$ & $0(0.0)$ & \\
\hline Open dumping & $41(85.4)$ & $7(14.6)$ & \\
\hline Wastes dried and burnt & $39(100.0)$ & $0(0.0)$ & \\
\hline Wastes gathered and dried & $57(100.0)$ & $0(0.0)$ & \\
\hline \multicolumn{4}{|l|}{ Water source used } \\
\hline Borehole & $26(100.0)$ & $0(0.0)$ & \multirow{4}{*}{$10.1931 *(\mathbf{0 . 0 2 5})^{+}$} \\
\hline Community borehole & $66(100.0)$ & $0(0.0)$ & \\
\hline Community standpipe & $152(93.8)$ & $10(6.2)$ & \\
\hline In-house pipe borne & $68(100.0)$ & $0(0.0)$ & \\
\hline \multicolumn{4}{|l|}{ Municipality } \\
\hline AMA & $160(97.0)$ & $5(3.0)$ & \multirow{7}{*}{$11.6076(0.071)$} \\
\hline Bawku West & $31(100.0)$ & $0(0.0)$ & \\
\hline Bolgatanga & $28(93.3)$ & $2(6.7)$ & \\
\hline Kasena-Nankana East & $23(100.0)$ & $0(0.0)$ & \\
\hline LADMA & $21(87.5)$ & $3(12.5)$ & \\
\hline LEKMA & $11(100.0)$ & $0(0.0)$ & \\
\hline Weija-Gbawe & $38(100.0)$ & $0(0.0)$ & \\
\hline \multicolumn{4}{|l|}{ Pig management system } \\
\hline Free-ranged & $92(96.8)$ & $3(3.2)$ & \multirow{2}{*}{$0.0424(0.837)$} \\
\hline Confined & $39(97.5)$ & $1(2.5)$ & \\
\hline
\end{tabular}

${ }^{*}$ Significant at $p<0.05 ;{ }^{+}$Fisher's exact $p$ values (some cell frequencies $<5$ ). 
seroprevalence was recorded among pig farmers from Accra who practiced the free-ranged system (Table 2).

All the pig samples which showed serological evidence of infection to Taenia solium came from pigs selected from the Upper East Region (Figure 3).

From this study, the overall seroprevalence of Taenia solium among pigs in both Accra and the Upper East Region was $24.9 \%(61 / 245)$ (Table 3). There was a statistical association between location and seropositivity to Taenia solium among pigs $(p=0.005)$ (Table 3$)$. There was a strong statistical association between municipality and Taenia solium seropositivity in pigs $(p<0.001)$ (Table 3$)$.

There was also a statistical association between the pig management system employed by pig farmers and seropositivity to Taenia solium in pigs $(p=0.005)$ (Table 3 ).

3.2. Seroprevalence of Trichinella spp. among Humans. An overall seroprevalence of $0.31 \%$ was recorded among human respondents from this study, and there was no statistically significant difference between Accra and Upper East (Table 4).

From this study, the overall seroprevalence of Trichinella spp. among pigs in both Accra and the Upper East Region was $4.5 \%(11 / 245)$ (Table 5). There was no statistical association between location and seropositivity to Trichinella spp. among pigs $(p=0.336)$ (Table 5$)$. All the pig samples that tested positive for Trichinella spp. came from Bolgatanga in the Upper East Region. All the other locations recorded $0 \%$ seroprevalence. Again, all the samples that showed serological evidence of Trichinella spp. among pigs came from those pigs who were raised on the free-ranged system (Table 5).

\section{Discussion}

4.1. Seroprevalence of Taenia solium in Humans and Pigs. This study recorded very low prevalences for Taenia solium and Trichinella spp. in both humans and pigs across the study sites. This might be due to the fact that here in Ghana we mostly overcook our meat products. A Taenia solium seroprevalence of $3.1 \%$ in humans was recorded for this present study. This is markedly lower than the Taenia solium prevalence of $13.15 \%$ recorded by Bimi et al. [2] among the stool samples of people of Northern Ghana. However, the seroprevalence of $3.1 \%$ for Taenia solium was higher than another study in the Kintampo North Municipality where a prevalence of $1.1 \%$ was recorded among study participants [18]. The finding of this present study compares favourably with 3.1\% seroprevalence among respondents in West Cameroon [19]. In a systematic review of the seroprevalences of Taenia solium human cysticercosis, prevalences of $7.30 \%, 4.08 \%$, and $3.98 \%$ were recorded for circulating Taenia solium antigens for Africa, Latin America, and Asia, respectively [20]. Similarly, seroprevalences of $17.37 \%, 13.03 \%$, and $15.68 \%$ for Taenia solium antibodies were reported for Africa, Latin America, and Asia, respectively [20]. This present study recorded a seroprevalence of $24.9 \%$ for Taenia solium among pigs from the study sites. There was also an association between where the pigs came from and seropositivity to Taenia solium/cysticercosis. All the pigs which showed serological markers for Taenia solium came from the Upper East Region. This finding is not surprising because the Upper East Region is noted to have the worst sanitation coverage in Ghana with many open defecation sites. This means that these pigs have access to more open defecation sites. The seroprevalence of $24.9 \%$ recorded in this study is corroborated by a study in Uganda which found a slightly higher prevalence of $25.7 \%$ among pigs [21]. Permin et al. [16], however, found a lower Taenia solium prevalence of $11.7 \%$ when they researched pigs from the same region, i.e., the Upper East Region. Similarly, Bimi et al. [2] recorded Taenia cysts in $18.8 \%$ among pigs slaughtered for consumption in the Northern Region of Ghana. Through their stochastic model, Assana et al. [22] predicted the expected prevalence of Taenia solium porcine cysticercosis in Ghana at $25.6 \%$. In another study in pig slaughterhouses in Kumasi, Atawalna and Mensah [23] also found a porcine cysticercosis prevalence of $2.31 \%$, lower than the $24.9 \%$ recorded in this present study. Atawalna and Mensah [23] argue that porcine cysticercosis mostly is not related to clinical signs of disease and that most of the cases are detected during routine postmortem inspections during slaughter. Another study in the Adamawa state in Nigeria also recorded a similarly lower porcine cysticercosis prevalence of $3.2 \%$ [24]. However, a higher prevalence rate of $46 \%$ has been recorded among pigs in the Jos Metropolis in Nigeria [25]. Uganda has up to $45 \%$ of the pig population infected with cysticercosis in some villages, and cysticercosis prevalences in pigs in many countries stand at $10 \%$ [26]. Assana et al. [22] estimated that $73.31 \%$ of pigs in Ghana are at risk of Taenia solium porcine cysticercosis.

4.2. Seroprevalence of Trichinella spp. among Humans and Pigs. This is the first study in Ghana to report of positive serological evidence of Trichinella spp. infections in humans. Seroprevalences of $0.31 \%$ and $4.5 \%$ for Trichinella spp. in both humans and pigs, respectively, were recorded. Trichinellosis incidence rates of $0.02 \%$ and $0.04 \%$ have been found in studies in Ethiopia [27]. Studies around the world have recorded similarly low prevalences of Trichinella in humans. For example, De-La-Rosa et al. [28] found seroprevalences between $1.0 \%$ and $1.9 \%$ when they studied prevalence and risk factors associated with serum antibodies against Trichinella spiralis in Mexico. In rural communities in Chile, Contreras et al. [29] found a seroprevalence of 1.5\% among their study participants. Also, the seroprevalence of $0.31 \%$ recorded in this present study was lower than the $19.1 \%$ Trichinella seroprevalence recorded among humans in Northern Laos [30]. Okello et al. [31], however, found a higher seroprevalence of $58.6 \%$ for Trichinella spp. in human samples from Laos People's Republic. One major drawback in knowing the true prevalence of Trichinella infections is primarily due to the lack of accurate data on cases. De-La-Rosa et al. [28] postulated that trichinellosis in humans is likely present in subtle endemic form due to the fact that it can remain asymptomatic and also produces similar symptoms like typhoid fever which can lead to misdiagnosis especially in resource-poor settings. 


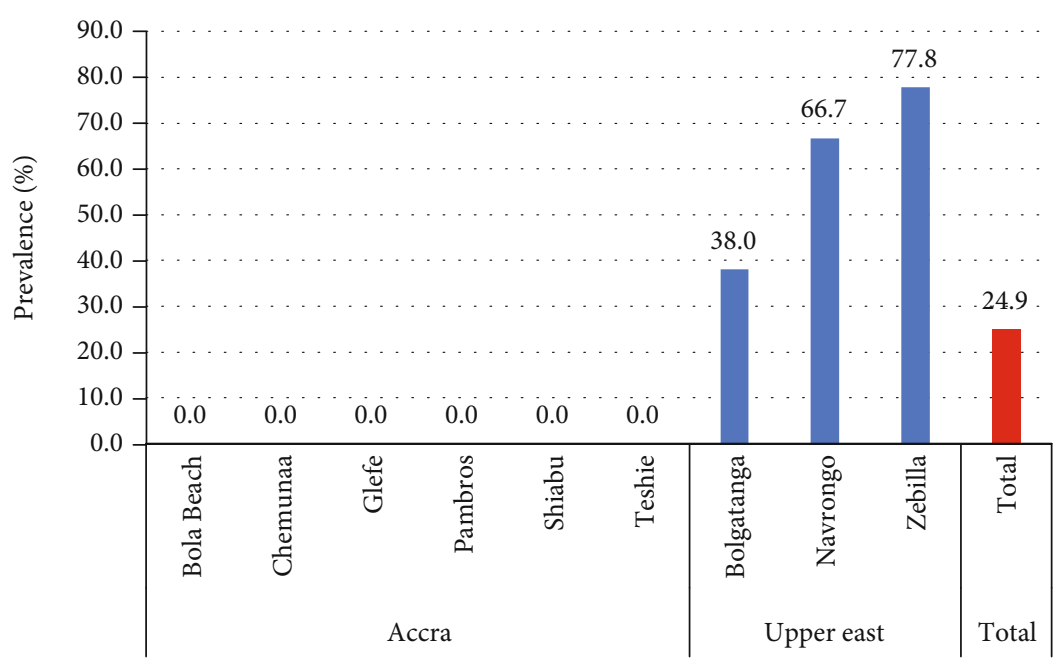

Figure 3: Prevalence of Taenia among pigs in Accra and Upper East by location.

TABle 3: Association between predictor variables and Taenia prevalence among pigs.

\begin{tabular}{|c|c|c|c|c|c|c|c|c|c|c|}
\hline \multirow{2}{*}{ Taenia solium (IgG) } & \multicolumn{3}{|c|}{ Negative } & \multicolumn{3}{|c|}{ Positive } & \multicolumn{4}{|c|}{ Total } \\
\hline & $N$ & Col $(\%)$ & Row (\%) & $N$ & Col (\%) & Row (\%) & $N$ & $\mathrm{Col}(\%)$ & Row (\%) & Prevalence (\%) \\
\hline \multicolumn{11}{|l|}{ Municipality } \\
\hline AMA & 58 & 31.5 & 100 & 0 & 0.0 & 0.0 & 58 & 23.7 & 100 & 0.0 \\
\hline Bawku West & 2 & 1.1 & 22.2 & 7 & 11.5 & 77.8 & 9 & 3.7 & 100 & 77.8 \\
\hline Bolgatanga & 85 & 46.2 & 62 & 52 & 85.2 & 38 & 137 & 55.9 & 100 & 38.0 \\
\hline Navrongo & 1 & 0.5 & 33.3 & 2 & 3.3 & 66.7 & 3 & 1.2 & 100 & 66.7 \\
\hline LEKMA & 22 & 12 & 100.0 & 0 & 0.0 & 0.0 & 22 & 9.0 & 100 & 0.0 \\
\hline Weija-Gbawe & 16 & 8.7 & 100.0 & 0 & 0.0 & 0.0 & 16 & 6.5 & 100 & 0.0 \\
\hline Total & 184 & 100.0 & 75.1 & 61 & 100.0 & 24.9 & 245 & 100 & 100 & 24.9 \\
\hline \multicolumn{11}{|l|}{ Pearson's Chi ${ }^{2}(5)=60.5772 p \leq 0.001$} \\
\hline \multicolumn{11}{|l|}{ Pig management system } \\
\hline Confined & 22 & 12 & 100 & 0 & 0 & 0 & 22 & 9.0 & 100 & 0.0 \\
\hline Free-ranged & 162 & 88 & 72.6 & 61 & 100 & 27.4 & 223 & 91.0 & 100 & 27.4 \\
\hline Total & 184 & 100 & 75.1 & 61 & 100 & 24.9 & 245 & 100.0 & 100 & 24.9 \\
\hline Pearson's Chi ${ }^{2}(1)=8.0130 p=0.005$ & & & & & & & & & & \\
\hline
\end{tabular}

TABle 4: Prevalence of Trichinella spp. among humans.

\begin{tabular}{lcccc}
\hline Location & $N(\%)$ & $\begin{array}{c}\text { Positive } \\
(\%)\end{array}$ & $\begin{array}{c}\text { Negative } \\
(\%)\end{array}$ & $\begin{array}{c}\text { Prevalence } \\
(\%)\end{array}$ \\
\hline $\begin{array}{l}\text { Accra } \\
\text { Upper } \\
\text { east }\end{array}$ & $238(73.9)$ & $1(0.4)$ & $237(99.6)$ & 0.4 \\
Total & $\begin{array}{c}322 \\
(100.0)\end{array}$ & $1(0.3)$ & $321(99.7)$ & $\mathbf{0 . 3 1}$ \\
\hline
\end{tabular}

Fisher' s exact $=0.3540 ; p$ value $=0.552$.

The $4.5 \%$ seroprevalence for Trichinella spp. recorded in this study came from pigs sampled from the Upper East Region of Ghana. Permin et al. [16] found 0\% prevalence when they studied cross-bred pigs from the same region.
Gamble and Bush [32] also found a very low prevalence of $0.013 \%$ of Trichinella spp. in pigs from the United States. Data on Trichinella spp. in pigs is lacking in Ghana. Only two studies from sub-Saharan African countries that found Trichinella-specific antibodies among domestic pigs have been published, reporting seroprevalences of $40 \%$ [33] and $11 \%$ [34]. Conlan et al. [30] also isolated Trichinella larvae from $2.1 \%$ of sampled pigs from Northern Laos, which is lower than the seroprevalence of $4.5 \%$ recorded in this present study. Another study found a higher Trichinella spp. seroprevalence of $23.4 \%$ among pigs in Laos People's Republic [31]. Similarly, a higher seroprevalence than what was recorded in this present study (6.9\%) was found among domestic pigs in Central and Eastern Uganda [35] and also in pigs (9.0\%) in endemic regions in Argentina [36]. Pozio [37] argues that the low prevalence of Trichinella spp. 
TABLE 5: Seroprevalence of Trichinella spiralis and predictor variables among pigs.

\begin{tabular}{|c|c|c|c|c|c|c|c|c|c|c|}
\hline \multirow{2}{*}{ Trichinella spp. } & \multicolumn{3}{|c|}{ Negative } & \multicolumn{3}{|c|}{ Positive } & \multicolumn{4}{|c|}{ Total } \\
\hline & $N$ & $\mathrm{Col}(\%)$ & Row (\%) & $N$ & Col (\%) & Row (\%) & $N$ & Col (\%) & Row (\%) & Prevalence \\
\hline \multicolumn{11}{|l|}{ Municipality } \\
\hline AMA & 58 & 24.8 & 100 & 0 & 0 & 0 & 58 & 23.7 & 100 & 0.0 \\
\hline Bawku West & 9 & 3.8 & 100 & 0 & 0 & 0 & 9 & 3.7 & 100 & 0.0 \\
\hline Bolgatanga & 126 & 53.8 & 92 & 11 & 100 & 8 & 137 & 55.9 & 100 & 8.0 \\
\hline Navrongo & 3 & 1.3 & 100 & 0 & 0 & 0 & 3 & 1.2 & 100 & 0.0 \\
\hline LEKMA & 22 & 9.4 & 100 & 0 & 0 & 0 & 22 & 9 & 100 & 0.0 \\
\hline Weija-Gbawe & 16 & 6.8 & 100 & 0 & 0 & 0 & 16 & 6.5 & 100 & 0.0 \\
\hline Total & 234 & 100 & 95.5 & 11 & 100 & 4.5 & 245 & 100 & 100 & 4.5 \\
\hline \multicolumn{11}{|c|}{ Pearson's Chi ${ }^{2}(5)=9.0792 ; p=0.106$} \\
\hline \multicolumn{11}{|c|}{ Pig management system } \\
\hline Confined & 22 & 9.4 & 100 & 0 & 0 & 0 & 22 & 9 & 100 & 0.0 \\
\hline Free-ranged & 212 & 90.6 & 95.1 & 11 & 100 & 4.9 & 223 & 91 & 100 & 4.9 \\
\hline Total & 234 & 100 & 95.5 & 11 & 100 & 4.5 & 245 & 100 & 100 & 4.5 \\
\hline \multicolumn{11}{|c|}{ Pearson's Chi ${ }^{2}(1)=1.1362 ; p=0.286$} \\
\hline
\end{tabular}

recorded among pigs in several studies despite millions of pigs tested yearly and people getting infected with Trichinella spp. means that the wrong pigs are usually tested. The emphasis should be placed on free-ranged scavenging pigs and not those reared under a controlled environment.

While the major risk factor for human trichinellosis is the consumption of contaminated meat or poorly cooked meat, the risk factors related to the presence of Trichinella spp. in pigs are the ingestion of infectious larvae by the scavenging behaviour of animals especially pigs. From the current study, all the pigs that showed serological markers of Trichinella spp. were those pigs reared on a free-ranged basis. This means that these pigs have access to open defecation sites, refuse dumps, and other contaminated sites as these pigs came from the Upper East Region in Ghana with the worst sanitation record. The low prevalences of Trichinella spp. in both humans and pigs recorded in this present study could be attributed to the lack of official data on pig slaughterhouses which might inform the prevalence. The situation has quite worsened due to home slaughter without veterinary supervision. Proper and adequate meat inspection is required to control the presence of Trichinella spp. in both humans and pigs. The pigs that tested positive for markers of Trichinella spp. all came from the Upper East Region. These communities in the Upper East Region are major supply conduits of pigs to Accra. This makes the role played by supervisory bodies like Veterinary Officers, Municipal/District Assemblies, and other allied regulatory bodies even more necessary. These pigs pass through several checks before they finally arrive in Accra.

\section{Conclusion}

Till date, no outbreak of Taenia solium and Trichinella spp. have been recorded in Ghana. Despite this, all the risk factors for their occurrence are present: increase in pork consumption, presence of free-ranging pigs, poor sanitation conditions in the country, and presence of open defecation sites. A One Health approach comprised of veterinary officers, environmental health officers, medical experts, and all aspects of social scientists is needed for zoonotic parasites of pigs like Taenia solium taeniasis and Trichinella spp. to be controlled.

\section{Data Availability}

The data used in this work is available upon request from the corresponding author.

\section{Conflicts of Interest}

The authors declare no conflict of interest in the publication of this research work.

\section{Authors' Contributions}

HOA conceived and designed the study. HOA developed the study tool, supervised data collection, and did the laboratory work and data analysis. AOM and ESD participated in study design and writing of the manuscript. LHOA supervised the laboratory analysis and writing of the manuscript. KEA performed the data analysis. DO and GOO supervised laboratory work and data collection. LB supervised the study tool development and editing of the final paper. All authors read and approved the final manuscript.

\section{Acknowledgments}

The authors would like to thank the University of Ghana Pan-African Doctoral Academy Thesis Completion Grant for sponsoring aspects of the doctoral work of the lead author. Also, the authors thank all the respondents who agreed to take part in the study and the National Public Health Reference Laboratory and the Noguchi Memorial Institute for Medical Research, University of Ghana. 


\section{References}

[1] A. N. Barnes, A. Davaasuren, U. Baasandagva, and G. C. Gray, "A systematic review of zoonotic enteric parasitic diseases among nomadic and pastoral people," PLoS One, vol. 13, no. 8, article e0201526, 2018.

[2] L. Bimi, A. Kankponang, and F. Anto, "Prevalence and risk factors of taeniasis in the Bunkpurugu-Yunyoo District of Northern Ghana," Journal of Bacteriology \& Parasitology, vol. 3, no. 1, pp. 1-4, 2012.

[3] H. R. Holt, P. Inthavong, B. Khamlome et al., "Endemicity of zoonotic diseases in pigs and humans in lowland and upland Lao PDR: identification of socio-cultural risk factors," PLoS Neglected Tropical Diseases, vol. 10, no. 4, pp. e0003913e0003916, 2016.

[4] E. Assana, M. W. Lightowlers, A. P. Zoli, and S. Geerts, “Taenia solium taeniosis/cysticercosis in Africa: risk factors, epidemiology and prospects for control using vaccination," Veterinary Parasitology, vol. 195, no. 1-2, pp. 14-23, 2013.

[5] A. Zoli, O. Shey-Njila, E. Assana, and J. Nguekam, "Regional status, epidemiology and impact of Taenia solium cysticercosis in Western and Central Africa," Acta Tropica, vol. 87, no. 1, pp. 35-42, 2003.

[6] J. Melki, E. Koffi, M. Boka, A. Touré, M.-K. Soumahoro, and R. Jambou, "Taenia solium cysticercosis in West Africa: status update," Parasite, vol. 25, p. 49, 2018.

[7] H. Carabin, A. S. Winkler, and P. Dorny, “Taenia solium cysticercosis and taeniosis: achievements from the past 10 years and the way forward," PLoS Neglected Tropical Diseases, vol. 11, no. 4, article e0005478, 2017.

[8] A. U. Edia-Asuke, H. I. Inabo, V. J. Umoh, C. M. Whong, S. Asuke, and R. E. Edeh, "Assessment of sanitary conditions of unregistered pig slaughter slabs and post mortem examination of pigs for Taenia solium metacestodes in Kaduna metropolis, Nigeria," Infectious Diseases of Poverty, vol. 3, no. 1, p. 45, 2014.

[9] U. C. Braae, C. F. L. Saarnak, S. Mukaratirwa, B. Devleesschauwer, P. Magnussen, and M. V. Johansen, "Taenia solium taeniosis/cysticercosis and the co-distribution with schistosomiasis in Africa," Parasites and Vectors, vol. 8, no. 1, pp. 1-14, 2015.

[10] D. N. Onah and S. N. Chiejina, "Taenia solium cysticercosis and human taeniasis in the Nsukka area of Enugu State, Nigeria," Annals of Tropical Medicine and Parasitology, vol. 89, no. 4, pp. 399-407, 1995.

[11] K. E. Banson, J. Nketsia-Tabiri, K. Anno, and E. K. Dagbui, "Economic and market analysis of swine rearing and pork production in Ghana," Journal of Life Sciences, vol. 8, no. 8, pp. 699-708, 2014.

[12] N. D. Coulibaly and K. R. Yameogo, "Prevalence and control of zoonotic diseases: collaboration between public health workers and veterinarians in Burkina Faso," Acta Tropica, vol. 76, no. 1, pp. 53-57, 2000.

[13] R. Ganaba, N. Praet, H. Carabin et al., "Factors associated with the prevalence of circulating antigens to porcine cysticercosis in three villages of Burkina Faso," PLoS Neglected Tropical Diseases, vol. 5, no. 1, pp. e927-e927, 2011.

[14] D. Adu-Gyasi, K. P. Asante, M. T. Frempong et al., "Epidemiology of soil transmitted Helminth infections in the middlebelt of Ghana, Africa," Parasite Epidemiology and Control, vol. 3, no. 3, article e00071, 2018.
[15] A. Roepstorff, H. Mejer, P. Nejsum, and S. M. Thamsborg, "Helminth parasites in pigs: new challenges in pig production and current research highlights," Veterinary Parasitology, vol. 180, no. 1-2, pp. 72-81, 2011.

[16] A. Permin, L. Yelifari, P. Bloch, N. Steenhard, N. P. Hansen, and P. Nansen, "Parasites in cross-bred pigs in the Upper East Region of Ghana," Veterinary Parasitology, vol. 87, no. 1, pp. 63-71, 1999.

[17] J. Charan and T. Biswas, "How to calculate sample size for different study designs in medical research?," Indian Journal of Psychological Medicine, vol. 35, no. 2, pp. 121-126, 2013.

[18] D. Humphries, E. Mosites, J. Otchere et al., "Epidemiology of hookworm infection in Kintampo North Municipality, Ghana: patterns of malaria coinfection, anemia, and albendazole treatment failure," American Journal of Tropical Medicine and Hygiene, vol. 84, no. 5, pp. 792-800, 2011.

[19] A. Nkouawa, A. R. Dschanou, R. Moyou-Somo, Y. Sako, and A. Ito, "Seroprevalence and risk factors of human cysticercosis and taeniasis prevalence in a highly endemic area of epilepsy in Bangoua, West Cameroon," Acta Tropica, vol. 165, pp. 116120, 2017.

[20] M. Coral-Almeida, S. Gabriël, E. N. Abatih, N. Praet, W. Benitez, and P. Dorny, "Taenia solium human cysticercosis: a systematic review of sero-epidemological data from endemic zones around the world," PLoS Neglected Tropical Diseases, vol. 9, no. 7, pp. e0003919-e0003920, 2015.

[21] Z. Nsadha, L. F. Thomas, E. M. Fèvre, G. Nasinyama, L. Ojok, and C. Waiswa, "Prevalence of porcine cysticercosis in the Lake Kyoga Basin, Uganda," BMC Veterinary Research, vol. 10, no. 1, pp. 239-239, 2014.

[22] E. Assana, J. Awah-Ndukum, A. P. Zoli et al., "Pig populations at risk of Taenia solium cysticercosis and subsequent financial losses in West and Central Africa," Revue d'élevage et de Médecine Vétérinaire Des Pays Tropicaux, vol. 72, no. 2, p. 73, 2019.

[23] J. Atawalna and S. Mensah, "Prevalence and financial losses associated with porcine cysticercosis in the Kumasi Metropolis of Ghana," International Journal of Livestock Research, vol. 5, no. 9, p. 21, 2015.

[24] A. Biu and J. Ijudai, "Prevalence and morphometric studies on porcine cysticercosis in Adamawa State, Nigeria," Sokoto Journal of Veterinary Sciences, vol. 10, no. 1, pp. 28-31, 2012.

[25] R. P. Weka and E. Ikeh, "Seroprevalence of cysticercosis and intestinal parasitism in pigs in Jos Metropolis," Journal of Animal and Veterinary Advances, vol. 8, no. 5, pp. 883-887, 2009.

[26] N. A. Mafojane, C. C. Appleton, R. C. Krecek, L. M. Michael, and A. L. Willingham, "The current status of neurocysticercosis in Eastern and Southern Africa," Acta Tropica, vol. 87, no. 1, pp. 25-33, 2003.

[27] K. Darwin Murrell and E. Pozio, "Worldwide occurrence and impact of human trichinellosis, 1986-2009," Emerging Infectious Diseases, vol. 17, no. 12, pp. 2194-2202, 2011.

[28] J. L. De-La-Rosa, J. G. Aranda, E. Padilla, and D. Correa, "Prevalence and risk factors associated with serum antibodies against_Trichinella spiralis_," International Journal for Parasitology, vol. 28, no. 2, pp. 317-321, 1998.

[29] M. C. Contreras, H. Schenone, L. Sandoval, and M. García, "Epidemiology of trichinosis in Chile. Prevalence study by immunodiagnostic reactions," Boletin Chileno de Parasitologia, vol. 49, no. 3-4, pp. 73-75, 1994.

[30] J. V. Conlan, K. Vongxay, B. Khamlome et al., "Patterns and risks of Trichinella infection in humans and pigs in Northern 
Laos," PLoS Neglected Tropical Diseases, vol. 8, no. 7, p. e3034, 2014.

[31] A. L. Okello, T. T. Tiemann, P. Inthavong et al., "Integrating market chain assessments with zoonoses risk analysis in two cross-border pig value chains in Lao PDR," Asian-Australasian Journal of Animal Sciences, vol. 30, no. 11, pp. 1651-1659, 2017.

[32] H. R. Gamble and E. Bush, "Seroprevalence of _Trichinella_ infection in domestic swine based on the National Animal Health Monitoring System's 1990 and 1995 swine surveys," Veterinary Parasitology, vol. 80, no. 4, pp. 303-310, 1999.

[33] H. A. Momoh, M. Bello, H. Inabo et al., "Prevalence and some risk factors associated with trichinellosis in backyard pig farms in Zaria, Nigeria," Tropical Animal Health and Production, vol. 45, no. 5, pp. 1149-1152, 2013.

[34] O. Adediran, E. Uwalaka, and J. Abiola, "Seroprevalence of Trichinella antibodies in pigs reared under different husbandry systems in southwestern Nigeria," Alexandria Journal of Veterinary Sciences, vol. 47, no. 1, p. 183, 2015.

[35] M. P. O. Baumann, R. Fries, K. Roesel et al., "First report of the occurrence of Trichinella-specific antibodies in domestic pigs in central and eastern Uganda," PLoS One, vol. 11, no. 11, pp. 1-16, 2016.

[36] S. M. Venturiello, G. J. M. Ben, S. N. Costantino et al., "Diagnosis of porcine trichinellosis: parasitological and immunoserological tests in pigs from endemic areas of Argentina," Veterinary Parasitology, vol. 74, no. 2-4, pp. 215-228, 1998.

[37] E. Pozio, "Searching for Trichinella: not all pigs are created equal," Trends in Parasitology, vol. 30, no. 1, pp. 4-11, 2014. 